\title{
THE COLPOSCOPIC DIAGNOSIS OF EARLY CERVICAL CARCINOMA
}

\author{
By J. D. ANDREw, M.A., M.B., B.CH., M.R.C.O.G. \\ Late Senior Registrar to the Department of Obstetrics and Gynaecology, St. Bartholomew's Hospital
}

The early diagnosis of carcinoma of the cervix uteri has engaged the attention of gynaecologists for many years. It has long been recognized that the earlier in its progress a carcinoma is detected, the greater is the chance of cure. The importance of this is underlined when it is appreciated that the diagnosis of a cancerous lesion at stage one rather than stage two increases the patient's chance of cure by nearly 25 per cent. The purpose of this communication is to consider the effectiveness of colposcopic examination of the cervix and to indicate its usefulness as an additional aid in the early diagnosis of cervical cancer.

\section{The Diagnostic Problem}

In the past decade much attention has been given throughout the world to cytological methods in the early diagnosis of carcinoma of the cervix. The chief importance of these methods lies in the ability to detect the non-ulcerated or pre-invasive lesions. The value of these techniques is now clearly recognized and their use is increasing in the gynaecological clinics in this country.

Cytological study of the cervix is easy to undertake as far as the clinician is concerned and is inexpensive to perform. As a means of screening out-patients it is perhaps ideal in its simplicity. The method, however, demands the attention of a competent cytologist whose training is to be measured in years rather than months, whose effective output is necessarily limited, and who requires the backing of considerable laboratory facilities and a well-trained technical staff. Furthermore, once a positive diagnosis of cancer cells is reported in a cervical smear by the cytologist, the responsibility for treatment is thrust squarely on the clinician's shoulders. His dilemma only becomes apparent in the early cases, and in particular with the so-called intra-epithelial carcinoma. He must confirm the cytological findings by biopsy and histological examination of the material before proceeding to treat the patient and it may be difficult, if not impossible, to decide from what area on the cervix such a biopsy should be made.
Attempts have been made to resolve this problem in various ways. The single biopsy of a ' suspicious' area may, or may not, give a positive result. Multiple random biopsies are more likely to be effective if the laws of chance are to be accepted. Both methods have been advocated, but it is obvious that neither is very satisfactory. Diathermy conization of the cervix provides a mass of charred material from which the histologist can hardly be expected to produce a satisfactory answer. Cold conization and ring biopsy are theoretically much more practical, but to be effective all the tissues removed should be serially sectioned, and this becomes a considerable labour even if only one in ro of the sections is studied histologically. A combination of the differentias surface biopsy of Ayre, with a punch biopsy of the affected quarted of the cervix, is attractive, buक practically difficult, and may still fail to localize $\overrightarrow{\mathrm{a}}^{+}$ small lesion.

To appreciate all these points is not to detract from the usefulness of the techniques which have been considered. It is important, however, to realize their limitations so that they can be overcome. The colposcope provides a way of effecting improvements.

\section{The Colposcope}

This instrument provides a means whereby a three-dimensional study of an illuminated surface can be made under various magnifications. It was originally devised by Hans Hinselman, of Hamburg, in 1925 for the study of the epithelium of the vulva, vagina and cervix. Many varieties of colposcope are now available, some of complex design, although the principle of the procedure remains a very simple one. $U p$ to the present day the colposcope has found little favour amongst British gynaecologists as a diagnostic instrument. The reasons for this are many, but foremost among them must be numbered the lack of satisfactory and easily manoeuvrable machines at a price that would enable those interested to study its practicability. Until recently suitable instruments have been difficult to obtain, but the position is now 
considerably improved and a variety are available, even if the demand in England is small.

Because of this shortage of instruments, very few British gynaecologists have been able to use the colposcope personally, and those who have visited clinics on the Continent and in America, where it is in use, have had only a fleeting opporitunty to study its possibilities. In the writer's own experience, such opportunities are of little use, as it takes some time to learn what are the normal appearances of cervical epithelium seen through the colposcope. It must be realized that the picture is very different from the normal unmagnified view to be obtained with a speculum. One does, in fact, see a very much bigger cervix, but it is not just an enlarged picture of the naked eye appearances. In fact, a whole new range of normal appearances has first to be learned and studied before the deviations from normal can be appreciated. In exactly the same way those undertaking cytoscopy, sigmoidoscopy, bronchoscopy and gastroscopy have to learn the normal appearances of the tissues studied before they are in a position to recognize, to understand and accurately to diagnose the conditions giving rise to abnormal appearances. More than this, the colposcope reveals epithelial changes which it is impossible to detect with the naked eye, and it is such changes which are of paramount importance in the detection of early cervical carcinoma.

\section{The Examination of the Patient}

It is necessary for this to be undertaken in the lithotomy position, as the various instruments available have been designed for use in that position, and a better exposure of the cervix is obtained by this method. Special chairs or tables have been designed for colposcopy which are not yet available in this country. They are more comfortable for the patient and the operator, but any means of placing the patient in the lithotomy position can be utilized.

A digital examination of the pelvic contents shculd first be made and then a speculum is introduced into the vagina in the usual manner and the cervix displayed. It will be found in practice that a bivalve speculum of Brewer's type is the most convenient.

Some authorities prefer to use two right-angled specula, which give greater access to the introitus. The colposcope is then adjusted in position and the inspection of the cervix begun.

\section{Colposcopic Procedure}

The bright light of the colposcope enables a detailed naked eye examination of the cervix to be made. If secretions obscure the view, they may be removed by dabbing with dry gauze-covered

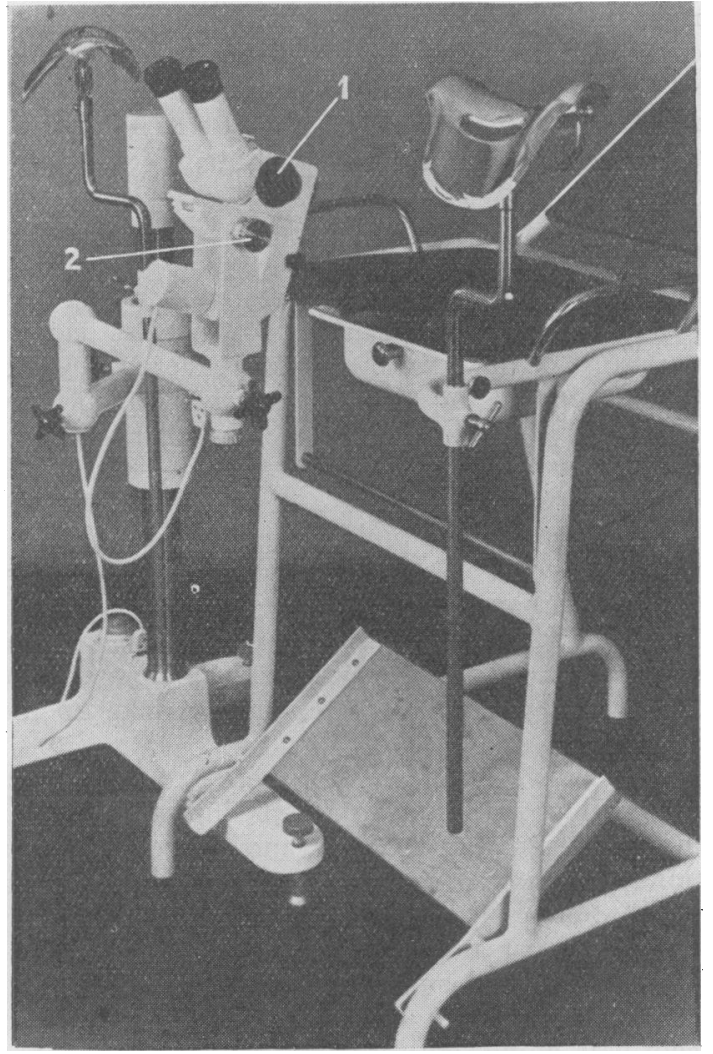

The photograph shows the Carl Zeiss Colposcope and a chair for colposcopy. The arrow (r) indicates the rotating magnification system. (2) Indicates the focusing knob. Both mechanisms are duplicated on the opposite side of the instrument.

cotton wool swabs. Care must be exercised to ensure that the underlying epithelium is not damaged.

It is usual to commence colposcopic examination at a magnification of ro times normal. More detailed study of specific areas at magnifications of 20 and upwards may be required later.

When this inspection has been completed the cervix is dabbed with a 2 per cent. solution of acetic acid, which precipitates the mucus overlying the endocervical epithelium and makes the gland openings and the papillae more obvious.

Subsequently the cervix is stained with iodine, as in Schiller's test for carcinoma, using a solution of pure iodine, ro parts; pot. iodide, 30 parts; aqua destillata, $35^{\circ}$ parts. This enables the extent of the atypical area of the epithelium to be more clearly defined and a pictorial record made. The whole examination can be undertaken and completed comfortably in five minutes and with increasing experience and dexterity it is possible to 
reduce this period to two or three minutes. In a clinic appropriately organized it is therefore possible to make this examination a brief part of the routine pelvic examination should it be desired to screen a large number of patients.

\section{Colposcopic Findings}

\section{(a) Normal}

To describe in detail the appearances which may be recognized through the colposcope lies outside the scope of this article and the reader is referred to the books and atlases already available by Ganse, Hinselman and Mestwerdt. Briefly, however, it is possible to distinguish three varieties of normal epithelium:

First, the smooth pink squamous epithelium of the portio can be seen which stains a mahogany colour with iodine.

Secondly, the glistening red mucous membrane of the endocervix appears as a papillary surface like bunches of grapes and does not take up the iodine stain at all.

Thirdly, at the junction of these two areas lies a marginal zone, where there is intermingling of the two types of epithelium, and where healing or transformation to squamous epithelium is taking place. Flame-shaped portions of young squamous epithelium can be seen extending into the grapelike mucosa and small islands of squamous and mucous epithelium appear. The former stain poorly if at all with iodine and are then of a yellowish colour.

\section{(b) Pathological}

Colposcopic examination reveals that atypical areas of squamous epithelium occur with considerable frequency. These are of great importance, as it is here that the cancerous change most probably develops.

The first condition to consider is leukoplakia. This is found, not uncommonly, as small white patches of horny growth and two varieties, ground and mosaic, occur. The former simply consists of an epithelium in which the keratinized covering has been lost or wiped off and appears as a welldemarcated area with punctuate red spots, which are the underlying capillary loops. The area stains yellow with iodine or remains pink. The mosaic pattern occurs when the condition affects the mucous glands of the endocervical mucosa, which are entirely, or almost entirely, filled with squamous epithelium of a different type. This has an appearance not unlike enclosed fields seen from the air and is sometimes called field formation.

A true erosion appears as a diffusely red area in which the acini of the mucous epithelium cannot be demonstrated when treated with acetic acid. As there are no squamous cells containing glycogen covering this surface, it remains red when it is treated with iodine.

When the atypical areas of epithelium already $\frac{\widehat{\alpha}}{\alpha}$ described continue to proliferate the demand for $c$ blood will increase and this process may continue $\Rightarrow$ for some time. The increased demand for blood results in an adaptation of the capillaries, which? grow wider and longer, and they may lose their normal shape. This adaptive hypertrophy may $\frac{\bar{p}}{\overrightarrow{7}}$

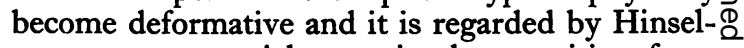
man as an essential stage in the transition from normal squamous epithelium to an established ${ }_{\vec{\rho}}^{\text {s }}$ squamous cell carcinoma of the cervix. These? changes can be detected quite clearly with the $\vec{\omega}$ higher magnifications available on the colposcope. $\stackrel{\omega}{\circ}$ When a frank carcinoma has formed, or a pre- 8 invasive cancer is present, these vascular changes 3 . become more marked. The capillaries are morew numerous, have bizarre shapes and are irregular in ${ }_{\omega}^{N}$ distribution, as is to be expected with the disorder $\omega$ of growth which has occurred. Furthermore, the i surface epithelium becomes irregular, it mayo ulcerate and it takes up no iodine stain. It is $\frac{\text { ? }}{2}$ findings of this nature which enable the colposcopicdiagnosis of pre-invasive or early invasive car- $Z$ cinoma to be made.

One word of warning must here be given: the 3 changes which occur with acute inflammatory lesions of the cervix may simulate some of the early appearances of carcinoma or of the atypiciclo varieties of squamous epithelium. A careful stuब⿶yo of the areas affected, and the use of staining solations, enables the differential diagnosis to be made, but confidence in the differentiation of these lesions is only to be acquired by experience. The discovery of the epithelial changes just described $\stackrel{\Omega}{\Omega}$ enables a diagnosis of early carcinoma or of pre- $\overrightarrow{\vec{z}}$ invasive carcinoma to be made at a time in the $\frac{3}{3}$ development of the growth before symptoms occur $?$ and before naked eye changes are apparent.

\section{Clinical Application}

It is evident from these observations that the colposcopic diagnosis of early invasive carcinoma of the squamous epithelium of the cervix is ai practical possibility. It is obvious, too, that as biopsy aimed at the affected area is made possibleo by this technique. This in itself is a considerable advantage and may well replace the more randomo methods described earlier.

Hinselman considers that the whole of theo affected area should be excised when the biopsyn is made, but this view is linked closely with theN subsequent treatment of the patient, which it is not
proposed to consider here.

It is not suggested that the colposcope should stand on its own in the early diagnosis of cervicalco carcinoma, but rather that it should be used in 
conjunction with other methods, such as cytological study, which are available to us. The earlier the lesions being dealt with, the more difficult the diagnosis becomes, and every aid is worthy of exploitation.

The possibilities of colposcopic study of the cervix have only been outlined. It is not only possible to diagnose early squamous cell carcinoma, but also in certain circumstances to detect adenocarcinoma of the endocervix. The method is also useful in eliminating benign lesions from the field of controversy and enables many conditions to be treated in the out-patient department without the necessity of admission for biopsy. The use of the instrument is not confined to inspection of the cervix alone, but can be extended to lesions of the vagina, vulva and urethra.

The major drawback to the use of the colposcope is the time taken in learning its capabilities. It is not an instrument for occasional or random use. Enough has been said, however, to indicate that the method is of real value and that effective trial should be given to this instrument in centres in this country where its usefulness can be properly studied.

\section{Acknowledgment}

I would like to acknowledge my debt to St. Bartholomew's Hospital, where the facilities to undertake this study were provided.

\section{BIBLIOGRAPHY}

GANSE, R. (1953), ' Kolpophotogramme zur Einfurhung in die Kolposkopie,' 2 vols., Akademie, Berlin.

HINSELMAN, H. (1954), ' Colposcopy,' W. Girardet, WuppertalElbertfield.

MESTWERDT, G. (1953), 'Atlas der Kolposkopi,' Gustav Fischer Verlag., Jena.

Bibliography continued from page 553-R. C. Percival, F.R.C.S., F.R.C.O.G.

\section{BIBLIOGRAPHY}

BEST, P. W. (1931), f. Amer. med. Ass., 97, 152 I.

CHARLWOOD, G. P., and CULINER, A. (1955), f. Obstet. Gynaec. Brit. Emp., 62, 555.

CUMMIN, R. C. (1955), Lancet, July 9, p. 68.

CURTIS, A. H. (1946), 'Textbook of Gynaecology,' 5th edition, Philadelphia, p. 560.

HIRST, B. C., and KNIPE, N. (1908), Surg. Gynaec. E' Obst., 7, 456 .
JEFFCOATE, T. N. A. (1955), f. Obstet. Gynaec. Brit. Emp., 62, 214 .

LAWSON, J. G., and CHOULER, F. J. G. (1955), Ibid., 62, 95 I LUND, J. (1955), Ibid., 62, 170.

MCINROY, R. A., and KELSEY, H. A. (I955), Ibid., 62, 428.

MURLEY, A. H. G. (1956), Lancet, June 23, p.994.

NOVAK, E. (1947), Gynaec. \& Obstet. Path., and edition, Philadelphia, p. 437 .

PEARSON, A. C. (1950), Proc. Roy. Soc. Med., 43, 443. 